\title{
XIII. On the connexion between the units of magnetism and electricity
}

\section{Prof. R. Clausius}

To cite this article: Prof. R. Clausius (1883) XIII. On the connexion between the units of magnetism and electricity , Philosophical Magazine Series 5, 15:92, 79-83, DOI: 10.1080/14786448308627316

To link to this article: http://dx.doi.org/10.1080/14786448308627316

$$
\text { 曲 Published online: } 08 \text { Jun } 2010 .
$$

\section{Submit your article to this journal $₫$}

Џ Article views: 4 
at each extremity of its swing ; but if it is naturally too quick, it will receive an outward impulse at each extremity. If its natural rate is sometimes too quick and sometimes too slow, according to temperature, it must therefore either gain or lose (practically it would probably always lose) an odd number of seconds when the temperature passes through the critical value.

\section{On the Connexion between the Units of Magnetism and} Electricity. By Prof. R. Clausius*.

TN a paper published in March of this yeart, I made some 1 remarks upon the systems of measures to be employed for the measurement of electric and magnetic quantities; and therein one point occurs which has given occasion to various objections-namely, the determination of the unit of magnetism in the electrostatic system. Some of these objections, which appeared in the June Number of the 'Philosophical Magazine,' I have already replied to in the August Number of the same journal. Since then, however, some further remarks, proceeding in part from parties worthy of high regard, have been made; so that I think it necessary again to introduce the subject.

In former times magnetism was regarded as an agent existing apart, independent of electricity. If that view were still adhered to, it would not be necessary, in establishing a system of measures for magnetism, to take into consideration the system employed for electricity. It is, however, Ampère's great merit, and, I believe I may add, one of the greatest advances made by physics, that an indissoluble connexion between electricity and magnetism has been demonstrated; so that we no longer need to consider magnetism a separate agent, but can regard all the forces usually called magnetic as electrodynamic.

For if we imagine, in a given magnet of measurable magnitude, each of the innumerable little magnets of which it consists replaced by a small electric current of which the definition is that the product of the current-intensity and the area round which the current flows is equal to the product of the length of the little magnet and the strength of its poles, or, differently expressed, equal to the magnetic momentum of the little magnet, then the totality of these small currents exerts

* Translated from a separate impression, communicated by the Author, from Wiedemann's Annalen, xvii. (1882) pp. 713-719.

+ Verhandl. des naturhist. Vereins der preuss. Rheinlande undWestfalens, xxxix. p. 105; Phil. Mag. June 1882,p. 381; Wied. Ann. xvi.p. 529 (1882). 
upon other electric currents or other magnets the same forces as the given magnet. Magnetism can thus, according to Ampère, be accounted for, without assuming the existence of a second agent besides electricity, from the existence of those electric currents.

According to this explanation, by the term Magnetism we are to understand only an electrodynamic conception; and hence, in every system of measures, the unit which is to be employed for measuring magnetism must be chosen in accordance with that conception-that is, so that the product of the unit of magnetism and the unit of length, and the product of unit current and unit area, become equivalent quantities. Therefore, if $[m]$ denotes the unit of magnetism, and $[i]$ the unit of current-intensity, and, further, $[\mathrm{L}]$ the unit of length and accordingly $\left[\mathrm{L}^{2}\right]$ the unit of area, the following equation must hold:-

from which follows

$$
[m \mathrm{~L}]=\left[i \mathrm{~L}^{2}\right] ; \cdot \cdot \cdots \cdot \cdot \cdot \cdot
$$

$$
[m]=[i \mathrm{~L}] \text {. }
$$

Now, since the unit of current-intensity is the intensity of a current in which, in the unit of time, the unit of electricity passes through a cross section, we have, if $[e]$ denotes the unit of electricity and $[\mathrm{T}]$ the time-unit, to put

$$
[i]=\left[e^{-1}\right] \text {; }
$$

by which the preceding equation is changed into

$$
[m]=\left[e \mathrm{LT}^{-1}\right] . . . . . .
$$

This equation expresses the relation between the units of magnetism and electricity corresponding to Ampere's explanation of magnetism. If we apply it to the electrostatic system of measures, and hence define the quantity [e], which in this case must, in order to distingujsh it from the electrodynamic unit of electricity, be written $\left[\rho_{s}\right]$, by the equation

$$
\left[e_{s}\right]=\left[\mathrm{M}^{\frac{1}{2}} \mathrm{~L}^{\frac{3}{2}} \mathrm{~T}^{-1}\right], \cdot \text {. . . . }
$$

in which $[\mathrm{M}]$ denotes the unit of mass, we get for the definition of the electrostatic unit of magnetism the following equation:-

$$
\left[m_{s}\right]=\left[\mathbf{M}^{\frac{1}{3}} \mathrm{~L}^{\frac{b}{2}} \mathbf{T}^{-2}\right] \text {. }
$$

It was this equation that gave rise to the above-mentioned objections, because it differs from Maxwell's, which reads as follows:-

$$
\left[m_{s}\right]=\left[\mathrm{M}^{\frac{1}{2}} \mathrm{~L}_{\frac{1}{2}}\right] \text {. }
$$

The way in which Maxwell arrived at his equation, as may be inferred from the connexion of his analyses, was based on 
the employment of an equation which differs from Ampère's with respect to its attitude towards the electrostatic and electrodynamic systems. For a distinction must be made between equations which belong exclusively to one of those systems and those which are applicable to both. Exclusively electrostatic or electrodynamic equations are those which represent an electrostatic or an electrodynamic force by the formula of mechanical force.

Now, as to Ampère's equation, it certainly resulted from the consideration of electrodynamic forces-that is to say, from the comparison of the forces exerted by a magnet and by an electric current; but it does not represent these forces either by the mechanical-force formula or by any other formula, but merely expresses that the magnet and the current are, in respect of the forces exerted by them, equivalent to one another. It is therefore not to be regarded as an exclusively electrodynamic equation; much rather, since it expresses only the relation which, according to Ampère, subsists between magnetism and electricity, to it must be ascribed, so far as Ampère's theory is at all recognized as correct, a general validity independent of the system of measures employed: and this justifies my application of it.

Maxwell's equation, on the contrary, was produced by representing an electrodynamic force, namely the force which a magnetic pole exerts upon an electric current, by the mechanical-force formula. It consequently bas in itself the character of an exclusively electrodynamic equation; and its employment for the determination of the electrostatic unit of magnetism cannot be acknowledged as justified.

On the reasons alleged by various English authors in support of this mode of determination I have already expressed myself in my above-mentioned article in the 'Philosophical Magazine,' and will not repeat what I have there said, but will confine myself to noticing some points which appear to me to furnish a standard for judging the mutually contrary modes of determination.

Maxwell's determination is not only carried out without regard to Ampère's explanation of magnetism, but is even in contradiction to it, as will readily be seen if the formula (6) above given for $\left[m_{s}\right]$, and at the same time formula $(4)$ for $\left[e_{s}\right]$, be inserted in equation (3), because then on the two sides there will be different expressions, and, indeed, expressions of different dimensions. And I must say that, if Ampère's explanation of magnetism, by which the great department of science embracing electricity, magnetism, and electromagnetism is so essentially simplified, were given up again, I should hold that a decidedly retrograde step had been taken. 
It is further to be remarked that, if once Ampère's explanation is abandoned and the electrostatic unit of magnetism is sought to be determined in another way, by employing one of the forces exerted by a magnetic pole, then Maxwell's formula is not the only possible one. I am induced to make this remark especially by a memoir recently published by Prof. Helmholtz*.

M. Helmholtz speaks of Gauss's units of electricity and magnetism, which are defined by the following propositions:(1) The unit of electricity is that quantity of electricity which at unit distance exerts the unit of force upon an equal quantity of electricity; (2) The unit of magnetism is that quantity of magnetism which exerts at unit distance the unit of force upon an equal quantity of magnetism. From these propositions result the equations :-

$$
\left.\begin{array}{rl}
{[e]} & =\left[\mathrm{M}^{\frac{1}{2}} \mathrm{~L}^{\frac{3}{2}} \mathrm{~T}^{-1}\right], \\
{[m]} & =\left[\mathrm{M}^{\frac{1}{3}} \mathrm{~L}^{\frac{3}{2}} \mathrm{~T}^{-1}\right] .
\end{array}\right\} . . . \quad . \quad .
$$

A system of measures based upon the simultaneous employment of these two equations M. Helmboltz calls the litherto employed electrostatic system, and in one place also the electrostatic magnetic system of Gauss.

Now I certainly do not believe that Gauss intended to make these two equations the foundation of one and the same coherent system of measures; I am much rather of the opinion that he regarded the electric and magnetic systems as two separate and independent systems, of which each would have one of the two equations for its basis. On the other hand, however, I willingly admit that, if we once abandon Ampère's explanation of magnetism, we can just as well combine these two equations into one system as those which underlie Maxwell's electrostatic system of measures.

We have consequently, including the system recommended by $M$. Helmholtz, three different electrostatic systems of measures, which agree perfectly with each other in relation to the purely electrical quantities, and differ only with respect to the unit of magnetism ; and here, for the sake of distinctness, the three formulæ representing the latter may be placed side by side.

\begin{tabular}{|c|c|c|c|}
\hline & Maxwell. & Helmholtz. & Clausius. \\
\hline$\left[m_{s}\right]$ & {$\left[\mathrm{M}^{\frac{1}{2}} \mathrm{~L}^{\frac{1}{2}}\right]$} & {$\left[\mathrm{M}^{\frac{1}{2}} \mathrm{~L}^{\frac{3}{2}} \mathrm{~T}^{-1}\right]$} & {$\left[\mathrm{M}^{\frac{1}{2}} \mathrm{~L}^{\frac{s}{2}} \mathrm{~T}^{-2}\right]$} \\
\hline
\end{tabular}

So far as we have to do only with the comparison of mag-

* Wied. Ann. xvii. p. 42 ; Phil. Mag. Dec. 1882, p. 43 ). 
netic quantities with one another, all three formulæ can serve equally well as formulæ for the unit of magnetism; but if we compare magnetic with electric quantities, the question arises, to what relations between magnetism and electricity do the different formulæ lead?

In the electrodynamic system, between the units of magnetism and electricity the following universally accepted equation holds :-

$$
\left[m_{d}\right]=\left[e_{d} \mathrm{LT}^{-1}\right] . \quad \cdot \quad \cdot . \cdot \cdot
$$

With this we will compare the equations which in the electrostatic system have to be formed, according to the three different conceptions of it, between the units of magnetism and electricity.

According to my conception we must put

$$
\left[m_{s}\right]=\left[e_{s} \mathrm{LT}^{-1}\right] \text {. . . }
$$

This equation has the same form as that which holds good in the electrodynamic system ; and consequently with this conception we get a definite relation between magnetism and electricity, independent of the system of measures employed. In the equations it is expressed that the unit of magnetism is a quantity having the dimension of a product of the unit of electricity and the unit of velocity. The occurrence of a velocity as a factor expresses that magnetism is to be placed in the same rank, not with resting (static), but with flowing (current) electricity, in accordance with Ampère's explanation.

According to Helmholtz's conception we must put

$$
\left[m_{s}\right]=\left[e_{s}\right] \cdot \text {. . . . . . . }
$$

This is quite another equation than that which is valid in the electrodynamic system; and I must confess I know not what idea is to be formed of the nature of magnetism if in one system of measures the unit of magnetism appears as a quantity having the same dimension as the unit of electricity, and in the other system as a quantity having the dimension of a product of the unit of electricity and the unit of velocity.

According to Maxwell's conception we must put

$$
\left[m_{s}\right]=\frac{\left[e_{s}\right]}{\left[\mathrm{L}^{\prime} \mathrm{I}^{-1}\right]} \cdot \text {. . . . . . }
$$

This is likewise a different equation from that current in the electrodynamic system. Instead of a product of the electricity- and velocity-units, here is a fraction having the electricity-unit for the numerator and the velocity-unit for the denominator; and the question again arises, What idea of the nature of magnetism is to be formed from two expressions so different?

Bonn, September 1882. 